\title{
BRINGING REALITY INTO THE CLASSROOM
}

\author{
André Heck
}

AMSTEL Institute, University of Amsterdam

Technology offers ample opportunities to bring reality into the classroom. Students and teachers nowadays have many tools to work in an authentic way with real data in mathematics and science education. However, much research and development is still needed to create a consistent learning trajectory out of the many exciting single activities. Some examples are presented, in particular with regard to video and image analysis, modelling, and simulation. In addition, authentic research projects of secondary school students are discussed.

\section{INTRODUCTION}

Coach is an activity-based, open computer learning environment that offers students and teachers a versatile set of integrated tools for data collection (through measurement with sensors or collection of data on video clips and digital images), for control of processes and devices, for processing and analysing data, for construction, simulation, and validation of computer models, and for authoring of activities. It is the result of more than two decades of sustained research and development work at the AMSTEL Institute of the University of Amsterdam to improve mathematics, science and technology education at primary and secondary school level. It has been translated into several languages and is used in many countries. The CMA Foundation (www.cma.science.uva.nl) distributes the Coach environment, which is meant to:

- $\quad$ facilitate the students' construction of in-depth and integrated understanding of mathematics and science concepts and processes through inquiry;

- change the computer into an instrument to explore real-world phenomena;

- involve students in similar activities to what 'real' scientists engage in and thus lead to authentic mathematics, science, and technology learning. To this end, students are offered tools for collecting, visualizing, processing, and analysing data, and for creating computational models and animations;

- be universal and applicable at many levels of education, in several curricula, and in various types of instruction, and be adjustable by teachers to their students' abilities.

I refer to (Heck, Kędzierska \& Ellermeijer, 2009) for a review of the principled design and implementation of Coach 6 . Here I discuss the opportunities of a computerbased approach to practical, investigative work in the mathematics and physics domain. I present some examples of activities involving measurements with sensors, video analysis, and modelling to study real-world phenomena in an inquiry approach. 


\section{MEASUREMENTS WITH SENSORS}

In a measurement activity with sensors, students gain insight into setting up an experiment and collecting data with sensors that are connected through an interface with a computer or directly attached to a hand-held device. This understanding of doing an experiment helps students to process and analyse the data, and to interpret the graphical representations of the data. It helps even more because the data are dynamically linked with different representations such as graphs, tables, meters, and digital values during and after the measurement, and because this measurement can be replayed on the computer screen as many times as wanted. Experiments are quite easily set up (by dragging and dropping sensor icons on the virtual interface panel on the computer screen or by automatic sensor recognition) with a variety of interfaces supported and a large library of calibrated sensors (for temperature, light intensity, sound level, etc.) available. Experimenters can select an appropriate measurement method (time-based, event-based, or manual measurement, with or without triggering) and a useful measurement setting (duration and sample rate). Students can also combine sensor-based data collection with synchronous video recording of the experiment done with a webcam. Heck and van Dongen (2008) presented a nice example of this feature of the Coach environment, concerning a high school student's research project on gait analysis.

The above description could give you the impression that students must do all the things mentioned to carry out a measurement activity with Coach, but this is not true. For young pupils, everything may be prearranged in such way that they cannot (accidentally) modify the activity. More experienced students may get access to measurement settings or the choice of sensors. Senior students may get maximum freedom of work for end-users, which is pretty close to the facilities for authors of Coach activities. This approach leads to a learning trajectory for doing measurements that works well in school practice (confer, van den Berg \& Ellermeijer, 2006). It is noted that the same tactics can also be applied in other types of Coach activities.

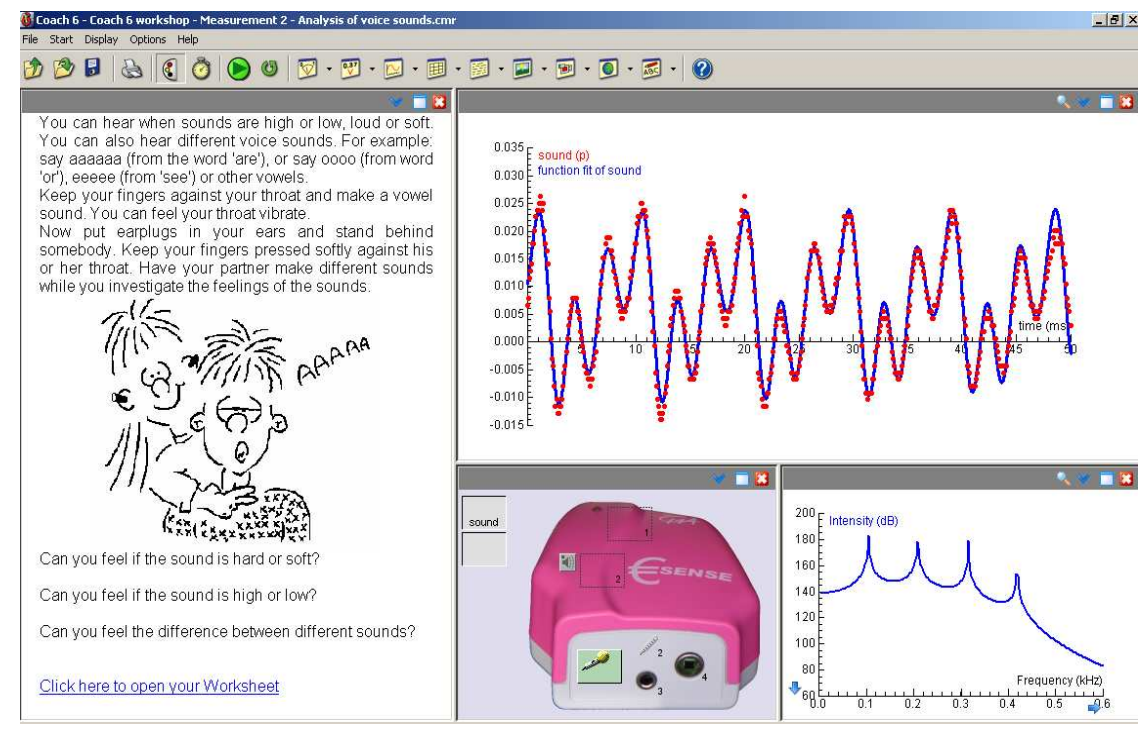

Figure 1. Measurement and signal analysis of voice sounds with the €Sense interface. 
Figure 1 shows a screen shot of the measurement (red dots) and the signal analysis (blue graph) of the voice sound 'eeh' recorded with the €Sense interface, which is mostly used at primary school or by beginners. A visual representation of the $€$ Sense interface is also present in the activity screen to make the experimental set-up clear to pupils. A text window is used for explanation and description of tasks. The diagrams illustrate that the sound signal is well described by a sinusoidal signal that consists of four frequencies. It goes without saying that the signal analysis is only done in an advanced course.

\section{DIGITAL IMAGE AND VIDEO ANALYSIS}

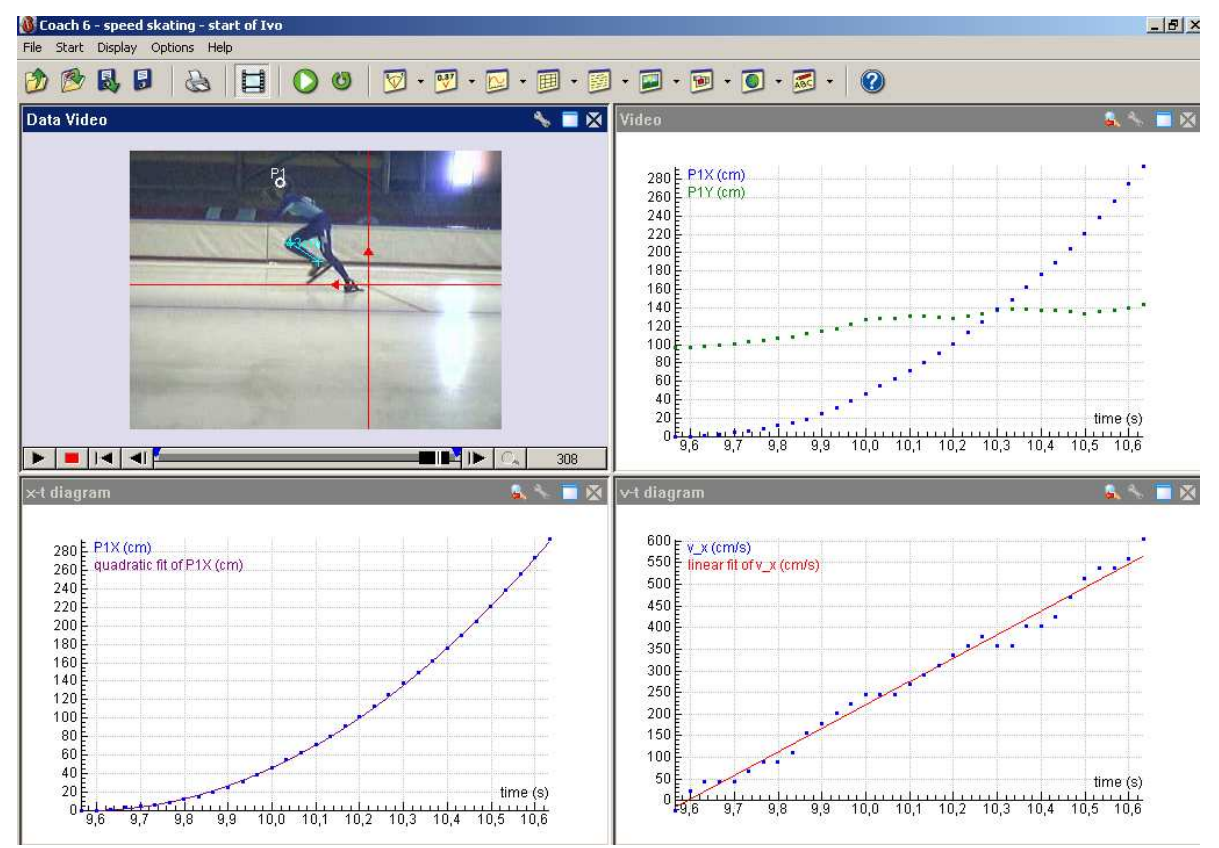

Figure 2. Screen shot of a video measurement of the start of a speed skater.

Figure 2 is a screen shot of a video measurement of the start of a young speed skater, which originates from the 2005 experimental computer exam in physics at the Dutch vocational stream level (see also, Boeijen \& Uylings, 2004) and has been part of a secondary school student's research project. The upper-left window contains the movie in which position data (after calibration of distance in the movie) have been collected by mouse clicking in the selected video frames on the head of the skater. In the upper-right diagram, the $x$ and $y$ coordinates of the skater's head are plotted against time. For a suitable choice of coordinate system and of starting time one gets the distance-time graph. A parabola fits the measured distance data in the lower-left diagram; this is clearly a good regression curve for the motion. The speed of the skater can be numerically computed for the measured data. The lower-right diagram contains the speed-time graph and a linear fit. This confirms the adequacy of the mathematical description of the skater's motion by a parabola. After this experimental modelling one could try to give a physics-based explanation.

It is considered an advantage of this predict-observe-explain (POE) approach to practical mathematics and science work suggested by Gunstone (1991, p. 69) that 
physics laws, mathematical formulas, graphs, approximation techniques, and so on, are not just compulsory items and/or a hobby of the teacher, but become in the eyes of students concrete, useful notions and methods that can be purposefully applied to come to grips with real-world phenomena. Secondary school students can develop in this way a broad range of skills that includes amongst others asking good questions, connecting a real world phenomenon with the world of mathematics and science, setting up an investigation or experiment, and collecting, representing, analysing, and interpreting information. Uylings and Heck (2005) reported about successful classroom experiments with video analysis activities at pre-vocational level. Heck and Holleman (2003) presented practical investigation tasks on human locomotion in which pre-university students (age 16-18 yr.) used the video analysis tool of Coach to collect and analyse real-time data about human gait in much the same way movement scientists do.

Some advantages of video and image measurement (see also, Laws \& Pfister, 1998), compared to measurements with sensors and traditional laboratory work, are:

- it is an easy, fast, broadly applicable, and attractive method of collection data in practical work for students who grow up with video technology and who can devise in this way their own projects;

- the experimental set-up is rather simple and a natural setting often suffices;

- $\quad$ simple mouse clicking and automated tracking replace the tedious work of manual recording of data and allow students to concentrate on the investigated phenomena. Data can be validated afterwards and corrected, if needed;

- $\quad$ processes that are too difficult or impossible to measure with sensors can be studied. The range of projects has been increased now that affordable high speed cameras have become available (See Heck \& Ellermeijer, 2009; Heck \& Uylings, 2009; Heck, Uylings \& Kędzierska, n.d.; Heck \& Vonk, 2009);

- $\quad$ students get acquainted with a major scientific research method, which is especially in biomechanics and movements science much used.

The tools for capturing and measuring video clips must be advanced and at the same time be understandable and easy-to-use for students and teachers in order to be advantageous in practice. Highlights of the video tool in Coach 6 are:

- $\quad$ an easy-to-use embedded tool for capturing of videos;

- standard image processing tools such as rotation and reflection of movies, and adjustment of brightness and contrast;

- $\quad$ correction of perspective distortion (also known as image rectification);

- $\quad$ automated point tracking.

The last two facilities have been added to Coach so that a user can deal with the following two common problems with video measurement: (i) the camera is not frontoparallel to the plane of motion; and (ii) manual video measurement is too timeconsuming and error-prone. Figure 3, taken from (Heck \& Vonk, 2009), illustrates the correction of perspective distortion in the context of moving coins on a flat surface. The image transformation is determined by mapping the four corners of the 
rectangular paper with a projective transformation to the corners of a rectangle in a new image (see the image to the right in Fig. 3). The frames in the video clip are all transformed such that the overlay becomes a rectangle. Scaling and translation of the rectified image are optional.

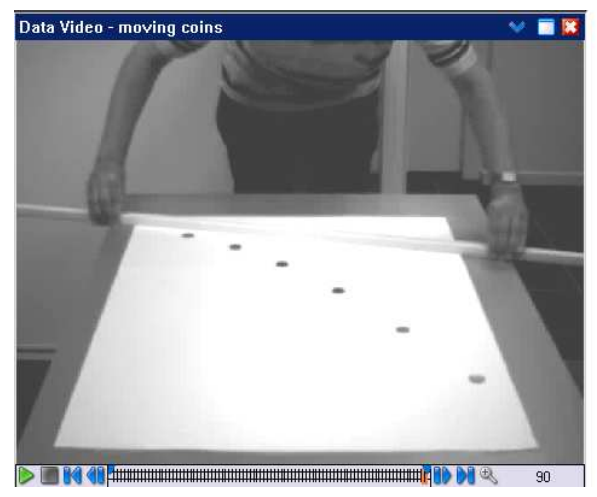

Figure 3.

(a)

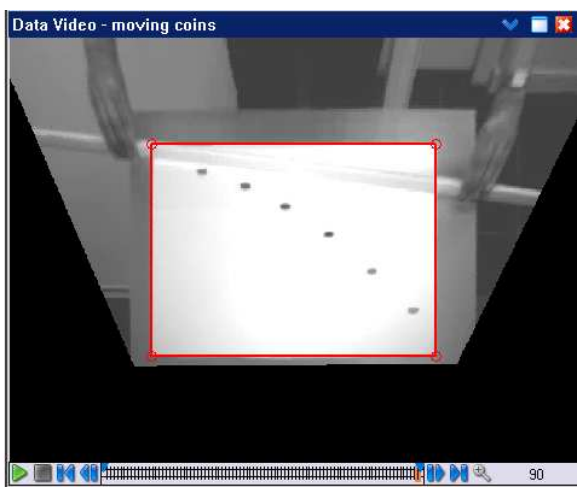

(b)

Video of moving coins before (a) and after (b) image rectification.

Figure 4, taken from (Heck, Ellermeijer \& Kędzierska, 2009), illustrates automated point tracking in the context of a bouncing table tennis ball, recorded with a highspeed camera at a frame rate of $150 \mathrm{fps}$. For each point of interest (here the centre of the ball), the user specifies at the start of the tracking process a template around this point that will henceforth be automatically matched in subsequent video frames. Matching takes place in a certain moving search area, the size of which is also userdefinable. In Figure 4 the search area around the currently measured point $\left(\mathrm{P}_{1}\right)$ is visible as a small rectangle.

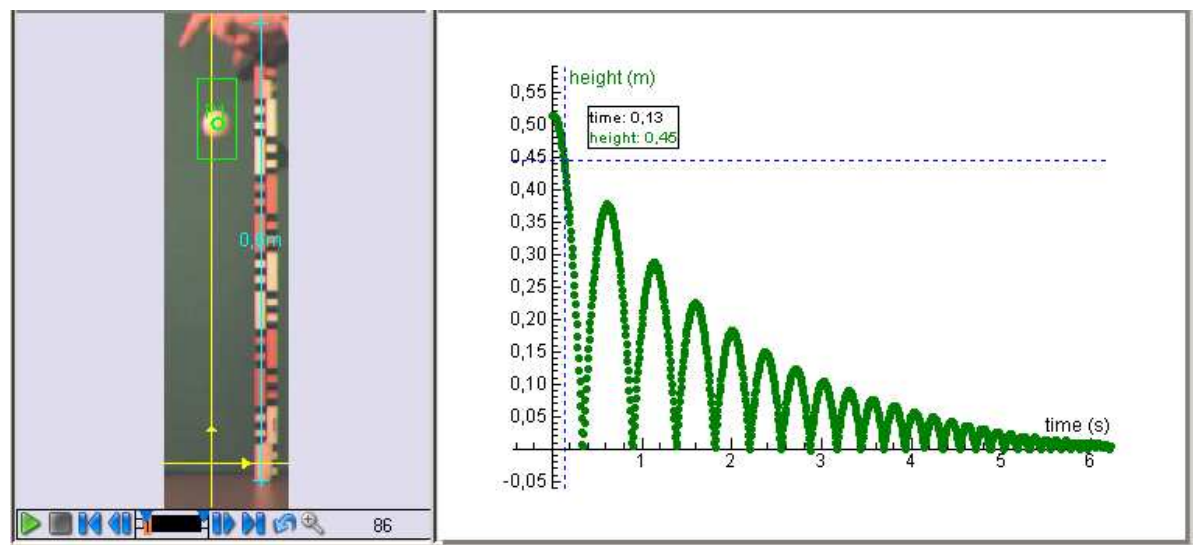

Figure 4. Video measurement of a bouncing table tennis ball via point tracking.

\section{MODELLING}

The modelling tool of Coach allows students to create and run numerical models, and to compare modelling results with experimental data. Three types of model editors are available: text-based, equations-based, and graphical. The first type of modelling is actually nothing else than programming in a computer language that is dedicated to mathematics, science and technology. The last two types of modelling are based on the system dynamics approach introduced by Forrester (1961) at MIT during the late 
1950s and early 1960s, and applied to education through a computer-based environment aimed at K-12 classes and beyond (Forrester, 1994). System dynamics is an approach to understanding the behaviour of complex systems over time. Central concepts are the use of feedback loops and the metaphor of stocks and flows, which is typical for a modelling system like STELLA (Richmond, 1985; Steed, 1992). The graphical mode of Coach provides a similar framework and easy-to-use interface to build up and visualise the structure of dynamical systems and the interaction between its elements. These systems are created with graphical elements such as a state variable (similar to the concept of stock), a rate of change (sum of all in- and outflows of a state variable), an auxiliary variable, and a constant (a parameter in the model); see, for example, Figure 5. Relationships between model variables are visualised by connectors and specified by mathematical formulas. The dynamics of the system is determined by the rates of change of state variables, which from mathematical point of view represent ordinary differential equations that are numerically solved in a simulation. Actually, Coach is a hybrid system that combines a classical system dynamics approach with event-based modelling for processes that change abruptly. This extends the set of realistic problems that can be solved by students without the need of sophisticated mathematics beyond their educational level. Examples are models of yoyos (Heck \& Uylings, 2005), bouncing balls (Heck, Ellermeijer \& Kędzierska, 2009), and quantitative pharmacokinetics (in particular, alcohol metabolism; see Heck, 2007). Computer modelling is viable at secondary school level!

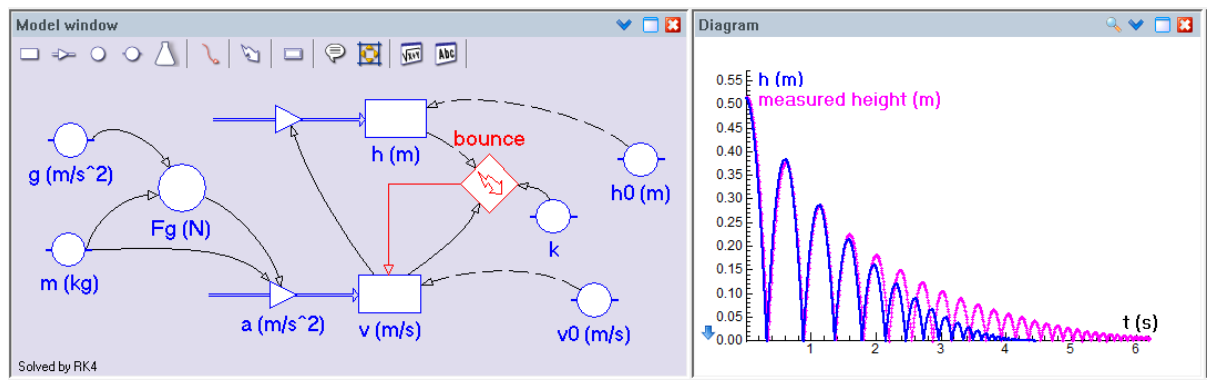

Figure 5. A limited model of a bouncing table tennis ball.

Figure 5 is a screen shot displaying a graphical model in Coach 6 that implements the bouncing table tennis ball by only taking into account gravity. When the ball is in the air, it is modelled by the system of differential equations $h^{\prime}=v, v^{\prime}=-g$, where $g$ is the acceleration of gravity, and $h$ and $v$ are the height and velocity of the ball, respectively. Height and velocity are state variables that are represented in the graphical model by rectangular boxes. The state variable $v$ is identified with the rate of change of $h$, represented in the graphical model by an inflow arrow. The acceleration $a$ is also represented by an inflow, corresponding to the rate of change of $v$. When the ball bounces, it is assumed that its velocity instantaneously changes direction and magnitude: $v_{\text {after }}=-k \cdot v_{\text {before }}$, where $k$ is called the coefficient of restitution. This sudden change is implemented as an event, with the following code behind the graphical element (the thunderbolt icon): Once $(h<=0)$ Do $v:=-k^{\star} v$ EndDo. The diagram in Figure 5 shows a height-time curve that has been calculated for the best guess of the 
coefficient of restitution $(k=0.865)$ using the 4th order Runge-Kutta method, which is one of the ODE solvers present in Coach. The measured height is shown as a background graph.

However, the result of the simulation is in this case rather disappointing: the model and the measurements match only for the first three or four bounces. The model also predicts a too short bounce time. At this point, students usually come up with various explanations such as "the laws of mechanics are not correct!", " $g$ changes with height", " $k$ is not constant", and "air resistance cannot be neglected." The last two arguments make sense. Here, I only show that incorporation of air resistance in the current model already makes a difference. In the extended model shown in Figure 6, the drag force $F_{\mathrm{d}}$ is assumed to be

$$
F_{d}=\frac{1}{2} \rho \cdot c_{d} \cdot A \cdot v^{2},
$$

where $\rho$ represents the density of air, $c_{\mathrm{d}}$ the drag coefficient, $A$ the cross-sectional area of the object, and $v$ the speed of the ball. The computer model and the measurements match now much better, although theory and reality still diverge rather quickly. It is only a first step in the repetitive process of improving the model that pre-university students successfully undertook in a practical investigation (see Heck, Ellermeijer \& Kędzierska, 2009).

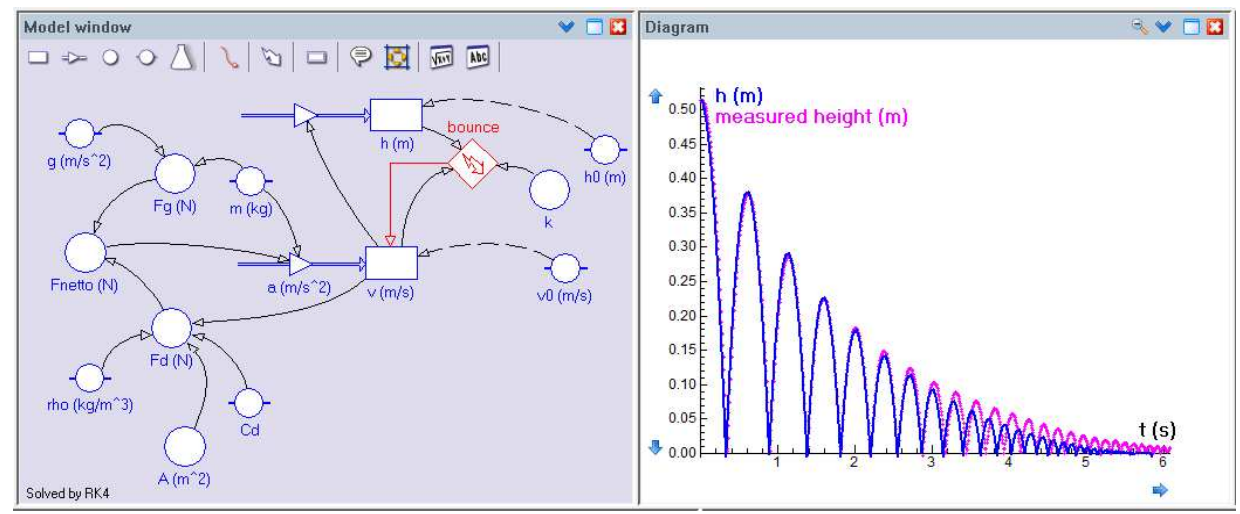

Figure 6. An improved model of a bouncing table tennis ball.

The short example of computer modelling of the bouncing ball gives a glimpse of the educational benefits of modern tools for students to construct and/or modify executable computer models of dynamic phenomena. Briefly, the benefits of a tool such as the one presented in this paper are:

- it can enhance students' theory building and scientific reasoning abilities;

- it allows students to study complex and realistic problems, not just limited to ideal situations. They may modify existing models and try out various modelling ideas, or they may construct new models from scratch.

- $\quad$ it offers students the opportunity to compare theoretical models with experimental results. Finding a suitable parameter value plays here a role as well;

- $\quad$ it gives students an idea of what mathematical modelling is and what its importance in science is. 


\section{GETTING A HEAD AROUND EXPONENTIAL DECAY}

The previous examples in this paper came all from cross-disciplinary investigative work of pre-university students in which laws of mechanics play an important role. Exponential laws are also common to many physical phenomena. Examples are cooling processes, radioactive decay, discharge of a capacitor, and so on. In this section I describe a fascinating example from real life for students to investigate with inexpensive means, namely, the collapse of the head of a beer poured into a glass. This example, which illustrates an entire investigation in a POE instructional approach to practical work, is part of the recently developed e-class on continuous dynamical systems for the optional Mathematics D course in the Netherlands. An eclass can be summarized as web supported instruction in a blended learning approach; for more details about the e-class concept, I refer to the descriptions of the eclasses on dynamical systems (Heck, Houwing \& de Beurs, 2009; Heck et al, 2009).

The subject of beer foam is not only fascinating for consumers of beer, who often judge a beer by foam, colour, and clarity unless they drink their beers directly from the bottle, but it is also for beer breweries which want to satisfy their customers by brewing beers with a stable but not excessive head, while taking preferences of consumers in different countries and regions into account (See, for example, Bamforth, 2000; Smythe, O’Mahoney \& Bamforth, 2002).

The experiment is simple and similar to the one described by Leike (2002) and by Hackbarth (2006): a natural beer pour into a glass with height measurements for beer and foam. Figure 7 shows a screen shot of a video analysis of the height of the beer (BeerH, defined as the height from the inside bottom of the glass to the beer/foam interface) and of the head of an alcohol-free beer (WetFoamH, defined as the height from the beer/foam interface to the top of the glass). The movie has been recorded within Coach via a webcam capturing 1 frame per 4 seconds. Some of the beer foam sticks to the side of the glass and this makes it difficult to get accurate measurements of the height of the head of the beer through automated point tracking. Nevertheless, this noisy data set will do for the purpose of the analysis of the experimental results.

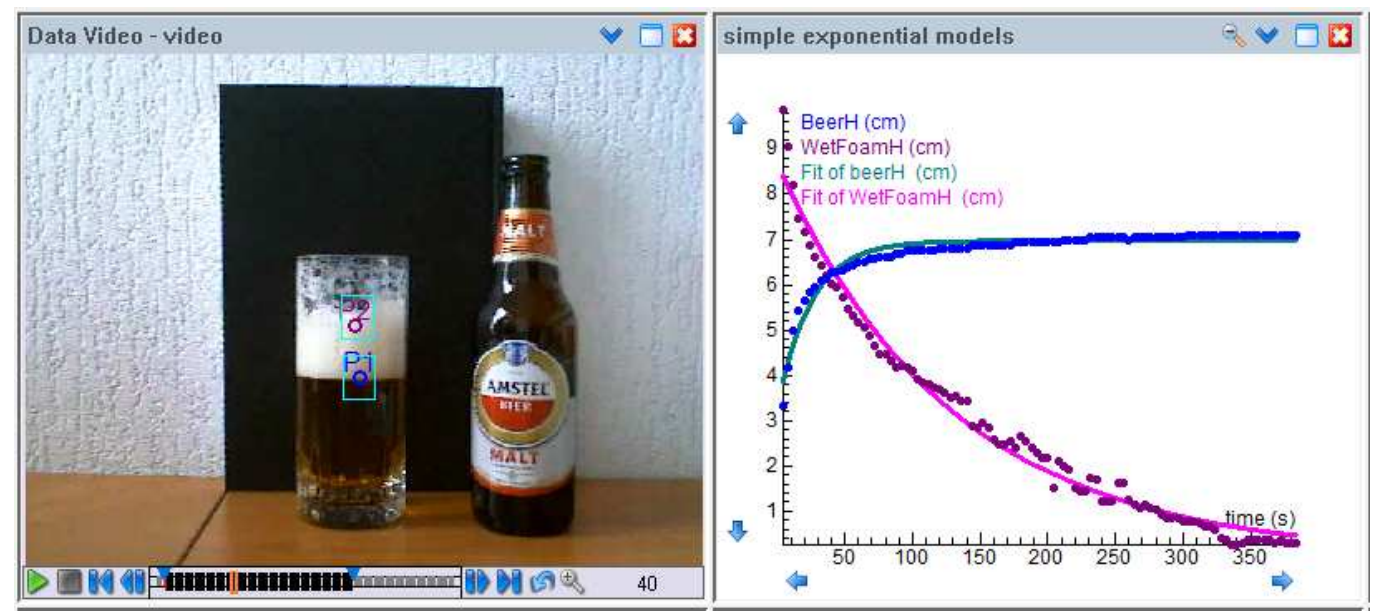

Figure 7. Screen shot of a video analysis of the head of an alcohol-free beer. 
The processes that play a role in the foam formation and retention are not simple and form the trick of the trade of beer brewing. The key physical events of foam formation, retention and adhesion are as follows (Lewis \& Bamforth, 2006): (1) bubble formation; (2) creaming; (3) drainage; (4) disproportionation; and (5) lacing. Beer is a supersaturated solution of gas. In the case of lager this is carbon dioxide, but in the case of ales it may be a mixture of carbon dioxide and nitrogen gas. During the beer pour there is condensation of the dissolved gasses and bubbles rise to the top of the glass forming the fine bubbled, white, and stable head of the beer with a high liquid fraction. The bubbles do not nucleate spontaneously, but need a nucleation site, which can be a particle in the beer, a scratch or imperfection on the glass (often especially designed beer glasses have roughened bases), or a preformed micro bubble. Creaming, also called beading, is the continuous formation of new bubbles. It not only is an appealing spectacle in beer, but also important because it replenishes the foam if it is sustained through the time for which the beer is in the glass. The main factors that influence the foam formation and creaming are temperature, the shape of the beer glass, the manner of pouring the beer into the glass, and the surface tension and liquid viscosity of the beer depending on the chemical composition. Drainage, that is, the downward flow of beer from the foam driven by gravity, begins as soon as the pour is complete. Dale et al (1999) found that a simple exponential law models the first phase of the decay of beer foam mass, which may take about 300 seconds (depending amongst other things on the type and temperature of the beer and the manner of pouring the beer into the glass) and accounts for 85-90\% loss of foam mass. In the diagram to the right in Figure 7, the best exponential decay curve for the measured head of the beer is shown, using the formula

$$
\text { WetFoamH }(\mathrm{t})=\mathrm{WetFoamH}(0) \cdot e^{-t / \tau} \text {, }
$$

where WetFoamH $(0)$ is the initial foam height at time $t=0$, and $\tau$ is a time constant called the lifetime. The graph matches the data well, except at the early stage, and the lifetime is 131 seconds with initial foam height equal to $8.7 \mathrm{~cm}$. In connection with this exponential decay, an exponentially decelerating growth curve fits the measured height of the beer. It is expressed by a function of the following type

$$
\operatorname{BeerH}(t)=c-a \cdot e^{-b \cdot t},
$$

where the best parameter values are $a=3.7(\mathrm{~cm}), b=0.04(1 / \mathrm{s})$, and $c=7(\mathrm{~cm})$.

As drainage proceeds, the foam structure changes from spherical to polyhedral bubble shapes and it shrinks. The concentration of polypeptide material in the foam increases and the foam coarsens through disproportionation (i.e., diffusion of gas from a small bubble to a larger adjacent bubble) and, as Ronteltap (1989) demonstrated, to a much lesser extent under normal condition through coalescence (i.e., merging of bubbles through the rupture of the film between them). This consolidation phase can also be modelled by exponential decay (Dale et al, 1999). Thus, the beer foam collapse is usually modelled through a biexponential model that consists of a fast and slow decay. Lacing, that is, foam adhering to the side of the glass, is ignored in this mathematical modelling. Beer foam is expected to last at least 5 minutes. 
I have spent many words on the processes that come into play during the formation and retention of the head of a beer because I want to make clear that a rather simple mathematical model like exponential decay can already be used to describe a complex real-life phenomenon. Refinement of this model will only be necessary when one wants to put the dots on the i's and add step-by-step more details to the model with the purpose of matching the model better with reality. This progressive aspect is common in mathematical modelling: first one simplifies the situation to such an extent that a simple model can be constructed. Hereafter one evaluates this model, preferably by comparing it with experimental data, and one adapts it if necessary. Adaptation of the model normally means that one makes the model more complicated by taking more factors, which cannot really be neglected, into account or by undoing some earlier simplifications. In my opinion, students should get ample opportunity to experience through investigative work the nature of modelling. I believe that by looking at various models of one and the same phenomenon a critical attitude of students is promoted. I shall illustrate this through further modelling of beer foam collapse.

In the particular example of the behaviour of beer poured into a glass, ignoring or separating the first stage of foam collapse improves the initial model. The diagram to the left in Figure 8 shows the exponential decay of the head of the beer and the exponentially decelerated growth of the beer height when only the measured data after 30 seconds have been taken into account in the regression methods. The regression curves match the measured data for that time period well, but this also means that the beer foam collapses at the early stage in reality faster than in the theoretical model; drainage of beer from the foam is then going faster than expected in the theoretical model. Correspondingly, the model of beer height overestimates the real height at the early stage. This can be understood because at the early stage the packing density of the bubbles in the foam is much less than later on in the experiment, which leads to a rapid loss of beer in the foam in the first 30 seconds following the pour.

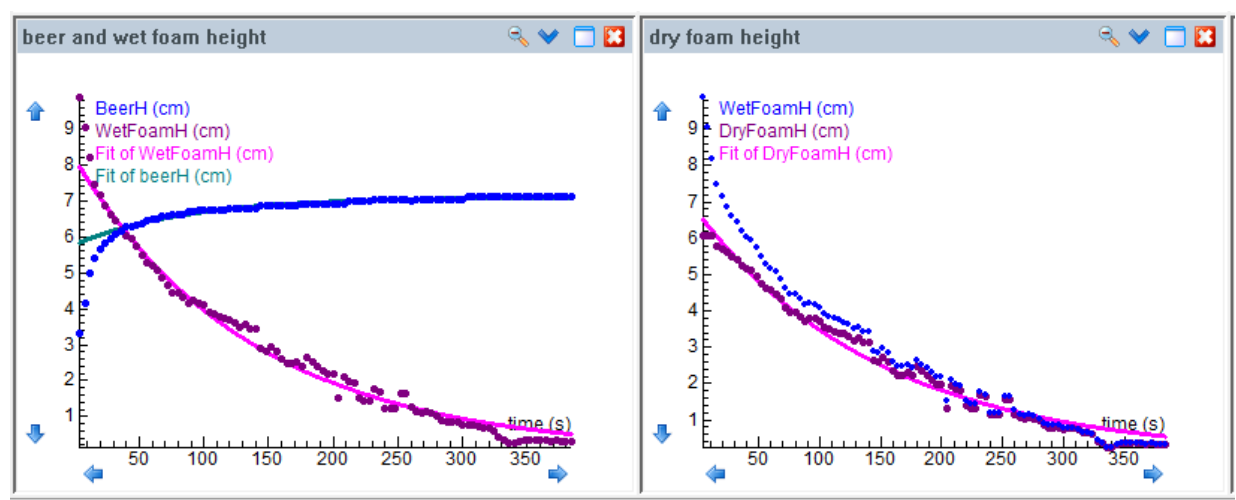

Figure 8. Screen shot of the exponential decay model of wet beer foam after 30 seconds (left) and of the exponential decay model of the dry foam height (right).

The beer model can also be improved by separating the wet part of the beer foam from the dry part of the foam, and by distinguishing between the contribution of 
drainage and condensation to the beer foam collapse. I follow Hackbarth (2006), who introduced dry foam height (DryFoamH) by

$$
\text { DryFoamH }=\text { WetFoamH }-(\text { FinalBeerH }- \text { BeerH })
$$

and showed that the dry foam height can be mathematically modelled well through exponential decay over the whole time period. The diagram to the right in Figure 8 illustrates that this also holds for the recorded pour of the beer in the given experiment. The lifetime of dry foam is then 153 seconds, which is as expected more than the lifetime found for wet foam. The initial dry foam height is $6.7 \mathrm{~cm}$.

Besides the experimental modelling of beer foam collapse through regression, one can also model the phenomenon in a system dynamics approach. Then, the aim is to set up a system of differential equations that reasonably describes the foam collapse and the increase of the beer height in the glass. The differential equations can be solved analytically or numerically on a computer. In my opinion, it is good when students learn both approaches of mathematical modelling and reflect on their strengths and weaknesses.

Below, I use the graphical modelling tool of Coach 6 to investigate the phenomenon of beer foam collapse by interrelated mathematical models. Each time I compare the results of a simulation run for suitable parameter values with the experimental data.

The exponential decay model of the head of the beer corresponds with the following ordinary differential equation:

$$
\frac{d}{d t} \operatorname{WetFoamH}(\mathrm{t})=-\frac{1}{\tau} \cdot \operatorname{WetFoamH}(t),
$$

where $\tau$ is the lifetime. The graphical representation in the modelling tool of Coach 6 is shown in Figure 9. The decay model with the most suitable values of initial height and lifetime matches the measured data rather well, except (as discussed before) for the first stage.

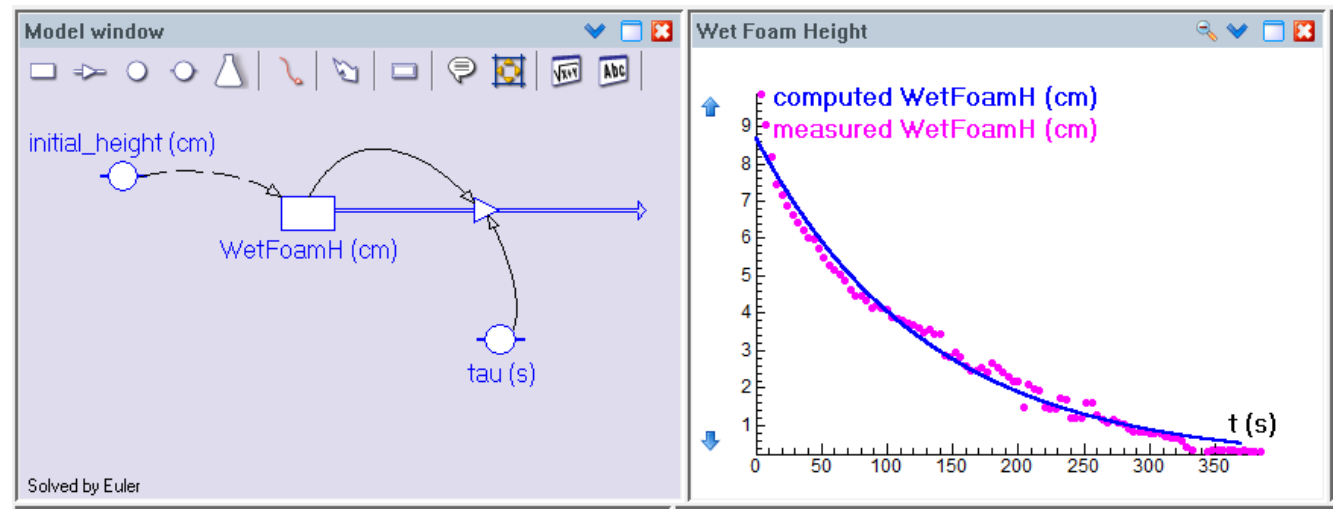

Figure 9. Screen shot of the exponential decay model of beer foam height.

The exponentially decelerated growth model of the beer height corresponds with the following ordinary differential equation:

$$
\frac{d}{d t} \operatorname{BeerH}(\mathrm{t})=b-a \cdot \operatorname{BeerH}(t)
$$


Figure 10 shows the solution curve for $a=0.04(1 / \mathrm{s})$ and $b=0.282(\mathrm{~cm})$, which are parameter values that give the best match with the measured data well all are taken into account. It has been noted before that this type of model works best if one ignores the beer collapse at the early stage of 30 seconds.

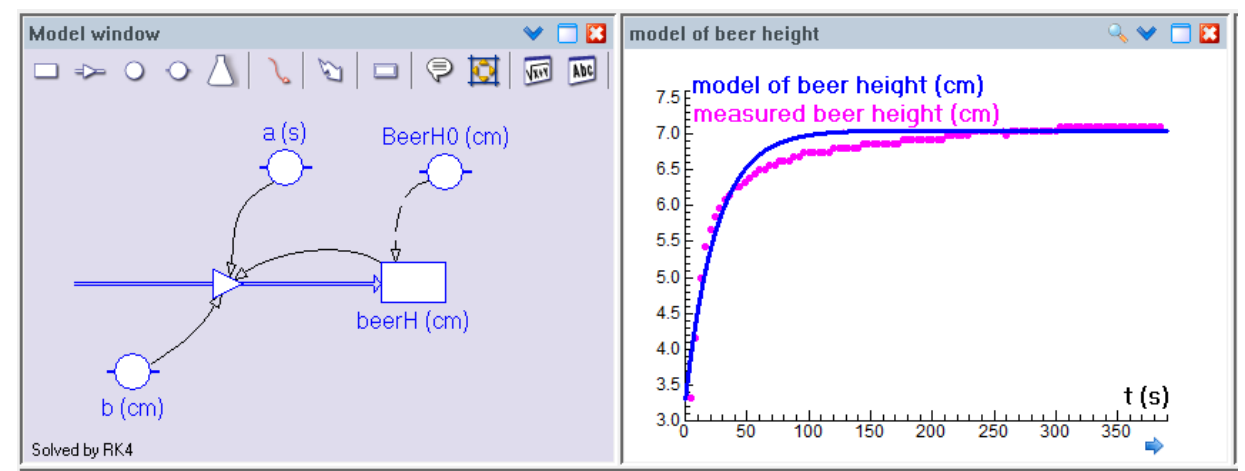

Figure 10. Screen shot of the exponentially decelerated growth model of beer height.

The beer foam collapse and the increase of the beer height are of course related to each other. It seems reasonable to model the two processes together through the following system of equations:

$$
\frac{d}{d t} \operatorname{WetFoamH}(t)=-\frac{1}{\tau} \cdot \operatorname{WetFoamH}(t), \frac{d}{d t} \operatorname{BeerH}(t)=-f \cdot \frac{d}{d t} \operatorname{WetFoamH}(t),
$$

where $f$ is the conversion factor that relates an amount of beer foam to an equivalent amount of liquid beer. Figure 11 is a screen shot of the corresponding graphical model, in which the Erlenmeyer symbol links the outflow of the foam height and the inflow of the beer height. Actually, it represents the following rewritten equations:

$$
\begin{aligned}
& \frac{d}{d t} \operatorname{WetFoamH}(t)=-\operatorname{Collapse}(t), \frac{d}{d t} \operatorname{BeerH}(t)=f \cdot \operatorname{Collapse}(t), \\
& \text { Collapse }(t)=\frac{1}{\tau} \cdot \operatorname{WetFoamH}(t) .
\end{aligned}
$$

The diagrams in Figure 11 have been obtained for the following parameter values:

$$
\tau=100(\mathrm{~s}), f=0.15 \text {, WetFoamH }(0)=10(\mathrm{~cm}), \operatorname{BeerH}(0)=5.65(\mathrm{~cm}) .
$$

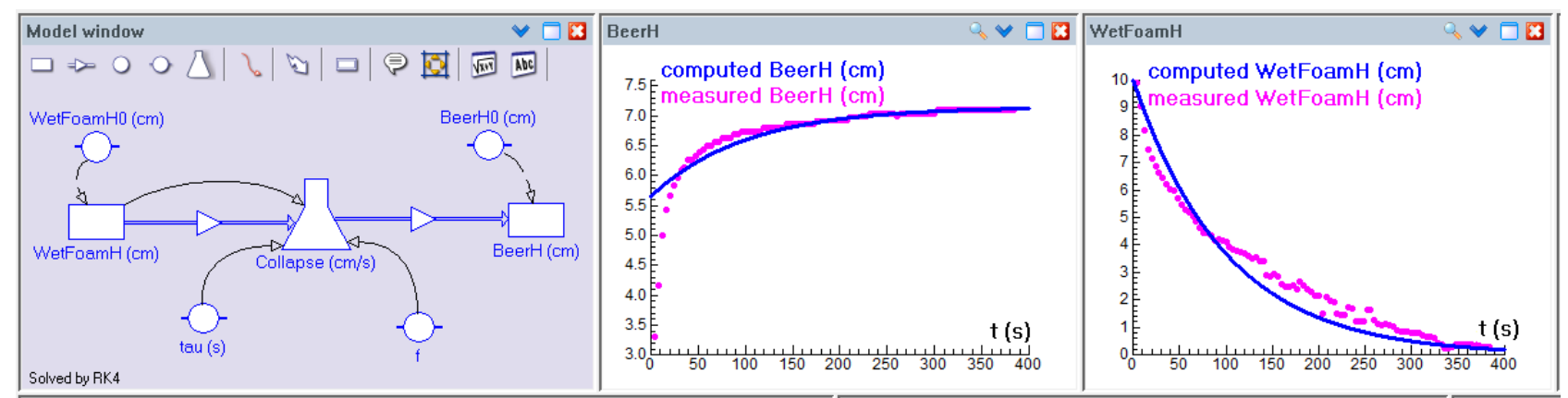

Figure 11. Screen shot of the coupled model of foam height and beer height.

A better match between experimental data and results from a theoretical model can be obtained by separating wet and dry foam height, just like Hackbarth (2006) did. It has already been noted that the dry foam height can be modelled well by an exponen- 
tial decay function. The wet part of the foam height (WetPartFoamH), defined as the difference between the beer foam height (WetFoamH) and the dry foam height (DryFoamH), turns out to be well modelled by a biexponential decay function in this particular beer experiment:

$$
\text { WetPartFoamH }(t) \approx 4.1 e^{-0.1 t}+1.1 e^{-0.01 t}
$$

The first term on the right-hand side describes the fast drainage at the early stage (with a lifetime of 10 seconds) and the second term is slow drainage later on (with a lifetime of 100 seconds).

The graphical model in Figure 12 corresponds with the following system of differential equations.

$$
\begin{aligned}
\operatorname{DryDecay}(t) & =\frac{1}{\tau_{\mathrm{dry}}} \cdot \operatorname{FoamH}(t), \quad \operatorname{WetDrainage}(t)=\frac{1}{\tau_{\text {wet }}} \cdot \operatorname{WetPartFoamH}(t), \\
\frac{d}{d t} \operatorname{DryFoamH}(t) & =-\operatorname{DryDecay}(t), \quad \frac{d}{d t} \operatorname{WetPartFoamH}(t)=-\operatorname{WetDrainage}(t), \\
\frac{d}{d t} \operatorname{BeerH}(t) & =f \cdot \operatorname{DryDecay}(t)+\operatorname{WetDrainage}(t) .
\end{aligned}
$$

Figure 12 shows the result when exponential decay of the wet part of the beer foam is assumed, that is, when a constant lifetime of the drainage $\left(\tau_{\text {wet }}\right)$ is assumed. This result cannot be improved much; one could only choose parameter values such that the model works well for the beer height only after the early stage of fast drainage.

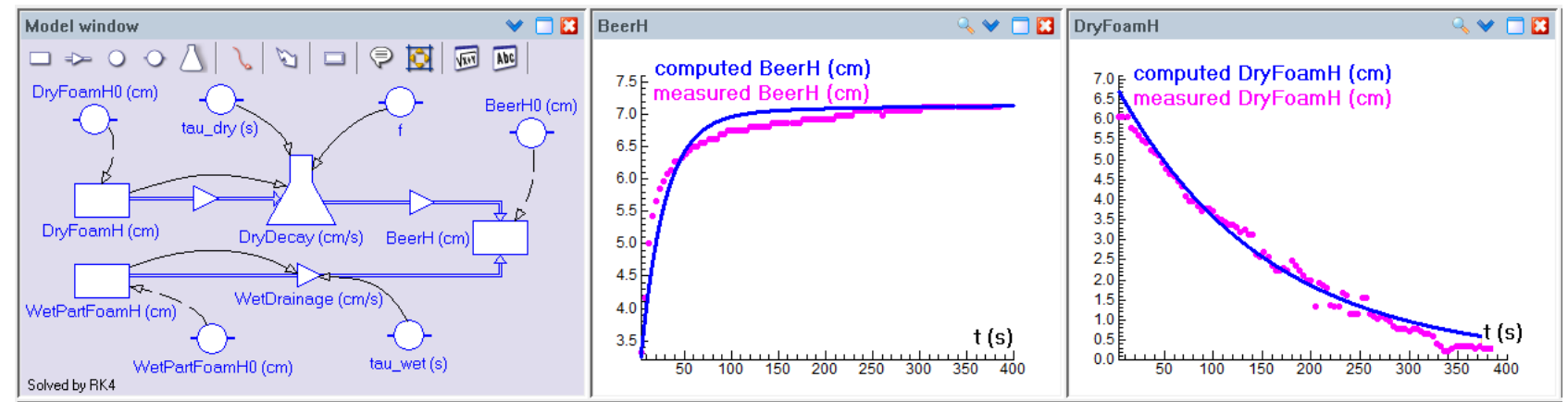

Figure 12. Screen shot of a coupled model of foam height and beer height with distinguished dry and wet parts of the foam.

The above model can be improved by using a suitable time-dependent function for $\tau_{\text {wet }}$. From the biexponential decay function of the wet part of the beer foam found earlier by regression, it is already known that such a function should be nearly constant and close to 100 for large values of time, should have values close to 10 in the early stage of foam collapse. By trial and error I constructed the following continuous function for $\tau_{\text {wet }}$, which more or less meets the stated criteria:

$$
\tau_{\text {wet }}=0.1+\frac{99.9}{1+1.2 e^{(2-0.05 t)}} .
$$


With this choice of the sigmoid function, a very good match of the computed model results and the experimental data is obtained. Figure 13 hardly leaves space for doubt. This makes the increase of the beer height also mathematically well modelled.

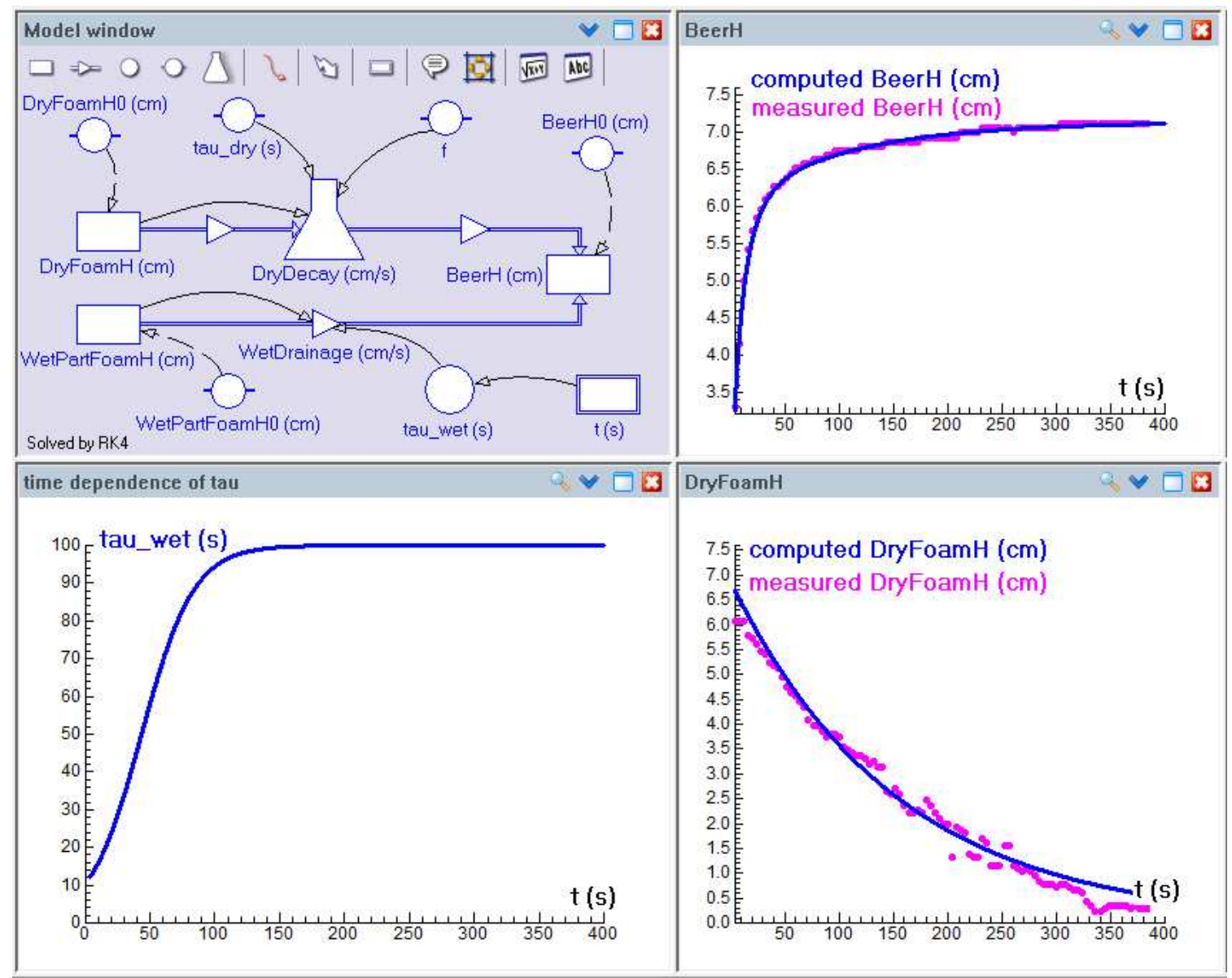

Figure 13. Screen shot of a coupled model of foam height and beer height with distinguished dry and wet parts of the foam, and with a time-dependent lifetime of the drainage of the foam.

The presented example of investigating the beer foam collapse and the other modelling examples in this paper illustrate that such authentic investigative activities offer students ample opportunity to practise their research abilities. The students learn to:

- formulate good research questions that guide them in the work;

- $\quad$ observe phenomena and discuss with peers about them;

- design and implement an experiment for collection of relevant data;

- $\quad$ apply mathematical knowledge and science concepts in new situations;

- $\quad$ find ways to use tools to solve problems at hand;

- $\quad$ construct, test, evaluate, and improve computer models;

- interpret and theoretically underpin results;

- $\quad$ get a scholarly attitude;

- reflect on own work and on work of others;

- collaborate with others in investigative work;

- $\quad$ report about and present own work. 


\section{CONCLUSION}

In mathematics and science, students are expected to develop a broad range of research abilities, which includes connecting a real world phenomenon with the scientific world, understanding the problems at hand and asking the right questions, making a project plan, designing and carrying out an experiment, and collecting, representing, analysing, and interpreting information. Students need ICT tools that make such investigation tasks feasible and that enable them to work at an appropriate level. In this paper I reported on a computer learning and authoring environment that provides students and teachers with a rich set of integrated tools, namely, Coach (Heck, Kędzierska \& Ellermeijer, 2009). This environment offers students opportunities to work like scientists with possibilities of collecting data with sensors, controlling experiments, measuring on video clips and digital images, processing and analysing data, exploring and creating executable model of dynamical phenomena, exploring and creating animations, and reporting of work. With these tools, students can carry out cross-disciplinary, authentic practical work in which the gap between mathematics and science learning at school and real life contexts is bridged through collection of real data by students. The authentic nature of such activities is interpreted as:

- the opportunity for students to work directly with high-quality, real-time data in much the same way professionals do;

- the resemblance of mathematics and science learning with practice. Students come in contact with present-day research work;

- the investigation are characterized as being challenging, complex and openended, cross-disciplinary, and not merely involving application of standard recipes, but also requiring a strong commitment to the tasks at hand, a broad range of abilities, including modelling and reflection skills.

The experiences with the students' research projects that have been cited in this paper justify the following conclusions:

- ICT enables and facilitates authentic, relevant, and high-quality projects for students in secondary education and beyond;

- students recognize the ICT possibilities immediately and on the basis of prior experience or preparation in lessons choose them for their practical assignments and research projects;

- $\quad$ secondary school students are able to do research in a way that resembles work of 'real' scientists.

\section{REFERENCES}

Bamforth, C.W. (2000). Perceptions of beer foam. Journal of the Institute of Brewing, 106(4) 229-238.

van den Berg, E., \& Ellermeijer, T. (2006). Science activities in a learning centre. In S.-O. Holmgren (Ed.), Proceedings of Science is Primary II (pp. 37-46). Stockholm: Royal Academy of Sciences. 
Boeijen, G., \& Uylings, P. (2004). Exams of tomorrow. In E. Mechlová (ed.) Teaching and Learning Physics in New Contexts, Conference Proceedings of GIREP 2004, Ostrava, Czech Republic (pp 153-154). University of Ostrava, Ostrava.

Dale, C., West, C., Eade, J., Rito-Palomares, M., \& Lyddiatt, A. (1999). Studies on the physical and compositional changes in collapsing beer foam. Chemical Engineering Journal 72(1) 83-89.

Forrester, J.W. (1961). Industrial Dynamics. Cambridge, MA: MIT Press

Forrester, J.W. (1994). Learning through system dynamics as preparation to the $21^{\text {st }}$ century. Keynote address for System Think and Dynamics Modeling Conference for K-12 Education. Concord Academy, Cord, MA. Retrieved (August 1, 2009) at http://sysdyn.clexchange.org/sdep/papers/D-4434-3.pdf

Gunstone, R.F. (1991). Reconstructing theory from practical experience. In B. Woolnough (ed.). Practical science: The role and reality of practical work in school science (pp. 6777). Milton Keynes: Open University Press

Hackbarth, J.J. (2006). Multivariate analysis of beer foam stand. Journal of the Institute of Brewing 112(1) 17-24.

Heck, A. (2007). Modelling intake and clearance of alcohol in humans. In E. Milková \& P. Prazák (eds.) Electronic Proceedings of the 8th International Conference on Technology in Mathematics Teaching (ICTMT8). University of Hradec Králové, Czech Republic.

Heck, A., \& van Dongen, C. (2008). Gait analysis by high school students. Physics Education 43(3) 284-290.

Heck, A., \& Ellermeijer, T. (2009). Giving students the run of sprinting models. American Journal of Physics (in press).

Heck, A., \& Ellermeijer, T., Kędzierska, E. (2009). Striking results with bouncing balls. In C.P. Constantinou \& N. Papadouris (eds.) Physics Curriculum Design, Development and Validation, Proceedings of the GIREP 2008 conference, Nicosia, Cyprus.

Heck, A., \& Holleman, P. (2003). Walk like a mathematician: an example of authentic education. In T. Trinadifillidis \& K. Hatzikiriakou (eds.) Proceedings of the 6th International Conference on Technology in Mathematics Teaching (ICTMT 6), University of Thessaly, Volos, Greece (pp. 380-387). Athens: New Technologies Publications

Heck, A., Houwing, H., \& de Beurs, C. (2009). An e-class in action: experiences with ICT intensive teaching and learning of discrete dynamical models at secondary school. Electronic Journal of e-Learning, 7 (1) 41-52.

Heck, A., Houwing, H., Val, J., Ekimova, L., \& Papageorgiou, P. (2009). Design and implementation of an e-class about continuous dynamical systems. Proceedings of the $6^{\text {th }}$ JEM Workshop. Retrieved (August 31, 2009) at: http://jem-thematic.net/en/node/1401

Heck, A., Kędzierska, E., \& Ellermeijer, T. (2009). Design and implementation of an integrated computer working environment. Journal of Computers in Mathematics and Science Teaching 28(2) 147-161. 
Heck, A., \& Uylings, P. (2005). Yoyo joy. In F. Olivero \& R. Sutherland (eds.) Proceedings of the 7th International Conference on Technology in Mathematics Teaching (ICTMT 7), Vol.2 (pp. 237-244). University of Bristol, UK.

Heck, A., \& Uylings, P. (2009). In a hurry to work with high-speed video at school. Physics Teacher (in press).

Heck, A., Uylings, P., \& Kędzierska, E. (n.d.). Understanding physics of bungee jumping (submitted).

Heck, A., \& Vonk, R. (2009). You must keep money moving. Physics Education 44(2) 188194.

Laws, P., \& Pfister, H. (1998). Using digital video analysis in introductory mechanics projects. Physics Teacher 36(5), 282-287.

Leike, A. (2002). Demonstration of the exponential decay using beer froth. European Journal of Physics 23(1) 21-26.

Lewis, M.J., \& Bamforth, C.W. (2006). Foam. In M.J. Lewis \& C.W. Bamforth (eds.), Essays in Brewing in Science (chapter 4, pp.28-42). New York: Springer Verlag

Richmond, B. (1985). STELLA: Software for bringing system dynamics to the other $98 \%$. Proceedings of the $3^{\text {rd }}$ Conference of the System Dynamics Society, Keystone, Colorado (pp. 706-718). Retrieved (August 1, 2009) at:

http://www.systemdynamics.org/conferences/1985/proceed/richm706.pdf

Ronteltap, A.D. (1989). Beer Foam Physics. PhD thesis, Agricultural University, Wageningen.

Smythe, J.E., O'Mahony, M.A., \& Bamforth, C.W. (2002). The impact of the appearance of beer on its perception. Journal of the Institute of Brewing 108(1) 37-42.

Steed, M. (1992). STELLA, a simulation construction kit: Cognitive process and educational implications. Journal of Computers in Mathematics and Science Teaching 11(1) 39-52.

Uylings, P., \& Heck, A. (2005). Capturing the real world in the classroom. In F. Olivero \& R. Sutherland (eds.) Proceedings of the 7th International Conference on Technology in Mathematics Teaching (ICTMT 7), Vol.2 (pp. 138-146). University of Bristol, UK. 